\title{
Hippocampal Subregion Transcriptomic Profiles Reflect Strategy Selection during Cognitive Aging
}

\author{
Garrett Smith, ${ }^{1}$ Asha Rani, ${ }^{1}$ Ashok Kumar, ${ }^{1}$ Jolie Barter, ${ }^{1}$ and Thomas C. Foster ${ }^{1,2}$ \\ ${ }^{1}$ Department of Neuroscience, McKnight Brain Institute, University of Florida, Gainesville, Florida 32610, and ${ }^{2}$ Genetics and Genomics Program, \\ University of Florida, Gainesville, Florida 32610
}

\begin{abstract}
Age-related cognitive impairments are associated with differentially expressed genes (DEGs) linked to defined neural systems; however, studies examining multiple regions of the hippocampus fail to find links between behavior and transcription in the dentate gyrus (DG). We hypothesized that use of a task requiring intact DG function would emphasize molecular signals in the DG associated with a decline in performance. We used a water maze beacon discrimination task to characterize young and middle-age male F344 rats, followed by a spatial reference memory probe trial test. Middle-age rats showed increased variability in discriminating two identical beacons. Use of an allocentric strategy and formation of a spatial reference memory were not different between age groups; however, older animals compensated for impaired beacon discrimination through greater reliance on spatial reference memory. mRNA sequencing of hippocampal subregions indicated DEGs in the DG of middle-age rats, linked to synaptic function and neurogenesis, correlated with beacon discrimination performance, suggesting that senescence of the DG underlies the impairment. Few genes correlated with spatial memory across age groups, with a greater number in region CA1. Age-related CA1 DEGs, correlated with spatial memory, were linked to regulation of neural activity. These results indicate that the beacon task is sensitive to impairment in middle age, and distinct gene profiles are observed in neural circuits that underlie beacon discrimination performance and allocentric memory. The use of different strategies in older animals and associated transcriptional profiles could provide an animal model for examining cognitive reserve and neural compensation of aging.
\end{abstract}

Key words: aging; hippocampus; pattern separation; reference memory; spatial discrimination; transcription

\section{Significance Statement}

Hippocampal subregions are thought to differentially contribute to memory. We took advantage of age-related variability in performance on a water maze beacon task and next-generation sequencing to test the hypothesis that aging of the dentate gyrus is linked to impaired beacon discrimination and compensatory use of allocentric memory. The dentate gyrus expressed synaptic function and neurogenesis genes correlated with beacon discrimination in middle-age animals. Spatial reference memory was associated with CA1 transcriptional correlates linked to regulation of neural activity and use of an allocentric strategy. This is the first study examining transcriptomes of multiple hippocampal subregions to link age-related impairments associated with discrimination of feature overlap and alternate response strategies to gene expression in specific hippocampal subregions.

Received Dec. 9, 2019; revised Apr. 8, 2020; accepted Apr. 30, 2020

Author contributions: G.S., A.R., A.K., and T.C.F. designed research; G.S., A.R., and J.B. performed research; G.S., J.B., and T.C.F. analyzed data; G.S. wrote the first draft of the paper; G.S., A.K., J.B., and T.C.F. edited the paper; G.S. and T.C.F. wrote the paper.

This work was supported by National Institute of Aging Grants AG037984, AG036800, AG049711, AG052258, and P30AG028740; and the Evelyn F. McKnight Brain Research Foundation. We thank Sebastian Conde, Nick Sarantos, Sophia Eikenberry, and Valentina Lavieri-Sosa for assistance in behavioral testing; and Dr. Brittney Yegla for assistance in data analysis and behavioral testing.

The authors declare no competing financial interests.

Correspondence should be addressed to Thomas C. Foster at Foster1@ufl.edu.

https://doi.org/10.1523/JNEUROSCI.2944-19.2020

Copyright $\odot 2020$ the authors

\section{Introduction}

Multiple brain regions exhibit similar transcriptional changes related to aging (e.g., immune response and lysosomal processes genes). In contrast, region-specific transcription is associated with performance of cognitive tasks that depend on the corresponding brain region (Prolla, 2002; Haberman et al., 2011; Masser et al., 2014; Ianov et al., 2016, 2017). For studies that examine the three main hippocampal subregions, impaired spatial memory is associated with robust transcriptional differences in regions $\mathrm{CA} 1$ and $\mathrm{CA} 3$ but limited differences in transcription within the dentate gyrus (DG) (Haberman et al., 2011; Masser et al., 2014; Ianov et al., 2017). The negligible correlation of DG transcription with cognitive impairment is somewhat surprising, 
given marked DG changes that occur with age, including decreased neurogenesis (Toda and Gage, 2018) and altered synaptic function (Gray and Barnes, 2015). This may be because studies exploring the relationship of transcription to cognitive impairment have not emphasized cognitive processes that depend on the DG (Ianov et al., 2017).

Experimental evidence supports a role for the DG in minimizing interference between overlapping features, such as those occurring in the same location or with perceptually similar objects. Animals sustaining a DG lesion exhibit impaired performance in a cheeseboard task, as demonstrated by lower accuracy to correctly identify a target object placed in close proximity to a lure object (Gilbert et al., 2001). In mice, localized NMDA receptor knock-out impairs the ability to distinguish two similar contexts or two identical beacons on a water maze task (McHugh et al., 2007; Bannerman et al., 2012; Taylor et al., 2013).

An age-related decline in the ability to distinguish objects as feature overlap increases or discriminate between the locations of two adjacent identical stimuli, often referred to a pattern separation, has been well documented in both humans (Bakker et al., 2008; Toner et al., 2009; Stark et al., 2010, 2013, 2015; Yassa et al., 2011; Holden et al., 2012; Ly et al., 2013; Reagh et al., 2014, 2018; Baker et al., 2016; Clark et al., 2017; Huffman and Stark, 2017) and animal models (Burke et al., 2010, 2011; Creer et al., 2010; Gracian et al., 2013; Wu et al., 2015; Johnson et al., 2016, 2017; Cès et al., 2018). Human functional imaging studies implicate the DG/CA3 complex in the age-related decline in ability to discriminate objects as feature overlap increases (Yassa et al., 2011; Bakker et al., 2012; Reagh et al., 2018).

Impaired ability to discriminate objects as feature overlap increases, associated with age, DG damage, or altered DG neurogenesis, can result in the use of different response strategies to maximize reward. Aging canines exhibit difficulties when discriminating between identical objects that are placed closer together but are still able to reach behavioral criteria (Snigdha et al., 2017). The ability to reach behavioral criteria was related to how quickly the animals acquired a new egocentric strategy to compensate for the impairment. In the case of DG lesions, rats were impaired in the ability to discriminate between adjacent locations signaled by two identical stimuli; however, rats were eventually able to acquire a spatial reference memory for the correct location (Gilbert et al., 2001; Morris et al., 2012). We hypothesized that impaired ability to distinguish two identical beacons on a water maze task will be measurable in middle-age rats compared with young, and that worse performance at middle-age will be associated with differential expression of genes (DEGs) within the DG. For middle-age animals, impairment was associated with age-related DEGs in the DG associated with synaptic function and neurogenesis. In addition, use of spatial reference memory in older animals, which were impaired for beacon discrimination, was associated with CA1 genes previously linked to superior spatial memory in aged animals and the use of an allocentric strategy.

\section{Materials and Methods}

Animals. Young (5 months, $n=22$ ) and middle-age (12 months, $n=30$ ) male Fisher 344 rats were obtained from the National Institute on Aging via the University of Florida Animal Care and Service facility. All rats were pair housed and maintained on a 12:12 h light cycle with ad libitum food and water access. All experiments were conducted in accordance with guidelines described by the US Public Health Service Policy on Humane Care and Use of Laboratory Animals and were approved by the University of Florida Institutional Animal Care and Use Committee.
Because of sex differences in behavioral pattern separation ability (Yagi et al., 2016) and variability in neurogenesis, transcription, and synaptic plasticity during the course of the rat estrus cycle (Crispino et al., 1999; Foster, 2005; DiCarlo et al., 2017), the current study focused only on male rats.

Apparatus. Behavioral experiments (Fig. 1A) were conducted in a circular black pool $\sim 169 \mathrm{~cm}$ in diameter. Water was maintained at $27^{\circ}$ $\mathrm{C}-29^{\circ} \mathrm{C}$ and a total depth of $\sim 20 \mathrm{~cm}$. The pool was located in a room with uniform black surroundings. Spatial cues were only present during spatial testing. The circular escape platform $(\sim 17.8 \mathrm{~cm}$ diameter $)$ was covered with a gray plastic mesh to facilitate gripping and was maintained $1 \mathrm{~cm}$ below the surface of the water. Beacons were comprised of white polystyrene foam balls $\sim 8 \mathrm{~cm}$ in diameter. The beacon above the escape platform was positioned on a black metal rod emerging from the center of the platform. The decoy beacon was positioned at a height equal to that of the beacon over the platform on an identically colored black rod that extended directly to the floor of the pool (Fig. 1B).

Cue discrimination training. Animals were allowed to habituate to the colonies of the McKnight Brain Institute for $7 \mathrm{~d}$ before beginning behavioral experiments. First, rats underwent a series of cue trials designed to train them to associate an escape platform with an overlying beacon (Foster, 2012). Rats were placed in the pool to swim freely for $30 \mathrm{~s}$, allowing for habituation to the pool. Rats were then subjected to 5 blocks of three trials each, with intertrial intervals of $20 \mathrm{~s}$ and interblock intervals of $\sim 15 \mathrm{~min}$. In each trial, rats were placed in one of four equally spaced start positions (N, S, E, and $\mathrm{W}$ ) and allowed up to $60 \mathrm{~s}$ to swim to an escape platform with an overlying beacon. Rats were gently guided to the platform if they were unable to find the platform within $60 \mathrm{~s}$. Rats remained on the platform for $\sim 20 \mathrm{~s}$ between trials. The positions of platforms and start locations were different for every trial within a block and were randomly determined in advance of the experiment. Between blocks, animals were returned to a holding cage with a warming fan placed near the cage.

Spatial discrimination testing. Forty-eight hours following cue testing (Fig. 1), rats were behaviorally characterized on a modified version of the beacon discrimination task as previously described (Bannerman et al., 2012). During the $5 \mathrm{~d}$ of discrimination testing, rats were placed in different positions within the perimeter of the pool containing two beacons: one with an underlying platform and the other in a diametrically opposite position of the pool with no underlying platform. Animals were able to navigate to the correct beacon using visual spatial cues placed on the walls around the pool. Each day of testing was comprised of two blocks of four trials with each trial starting point occurring in a pseudorandom sequence. Starting points occurred in one of six locations: two equidistant between the two beacons, two adjacent to the beacon overlying a platform $\left(\mathrm{S}^{+}\right)$, and two adjacent to the decoy beacon with no underlying platform. The $\mathrm{S}^{+}$and $\mathrm{S}^{-}$start positions were $45^{\circ}$ offset from the equidistant points relative to the center of the pool (Fig. 1B). A cohort of young $(n=10)$ and middle-age $(n=14)$ rats were first tested on the task with beacons separated by $73 \mathrm{~cm}$. A second cohort of young $(n=12)$ and middle-age $(n=16)$ rats were tested with beacons separated by $45 \mathrm{~cm}$ to allow assessments of increased task difficulty due to greater stimulus proximity.

One day after the last beacon discrimination test, animals were placed at a single equidistant start point in the pool and allowed to swim freely for $60 \mathrm{~s}$ with both beacons and the platform removed, but with all spatial cues unchanged, to determine whether animals had formed a spatial reference memory of the platform location (Fig. 1).

Statistical analysis of behavioral data. Statistics were computed using SPSS Statistics, version 26.0 (IBM). Data describing performance by day, start point, or block (in the case of cue trials) were analyzed by mixed ANOVA with within-subjects factors of day or start position and with between-subjects factors of age or beacon separation where applicable. Data from the first day of behavioral testing were excluded from analysis to correct for the time required to train rats to adapt to a second beacon. All post hoc tests were conducted with independent samples $t$ tests with 
Bonferroni correction. For measures of spatial reference memory, a discrimination index (DI) score was calculated for the probe trial using the formula: (time in goal zone - time in decoy zone)/(time in goal zone + time in decoy zone). The goal and decoy zones were defined as the circular areas within an $18 \mathrm{~cm}$ radius of the center of the goal and decoy zones, respectively. For instances in which Mauchly's test of sphericity was significant, Greenhouse-Geisser estimates of sphericity were used to correct degrees of freedom. Significant statistical effects and correlations were defined as those with $p<0.05$.

Tissue collection. Four to five days following the conclusion of spatial testing, rats were run on an abridged version of the discrimination task in which the positions of the beacon and platform were shifted $90^{\circ}$ from their position during beacon discrimination testing (Fig. 1). For these trials, each rat was placed at each equidistant start point (relative to the new beacon positions) twice for a total of four trials. Spatial cues for these tests remained in the positions used in initial beacon discrimination testing. These trials were done to stimulate hippocampal transcription of genes relevant to performance of the behavioral task. Approximately $1 \mathrm{~h}$ after concluding the fourth trial, rats were briefly anesthetized with isoflurane (Patterson Veterinary) and swiftly decapitated. The brains were then rapidly removed, and the hippocampi were dissected according to previously described methods (Lein et al., 2004; Zeier et al., 2011). The hippocampal regions DG, CA1, and CA3 were separated, placed in plastic tubes, and flash-frozen in liquid nitrogen. All samples were stored at $-80^{\circ} \mathrm{C}$ until processed. Precision of the dissection technique was verified by comparison of genes highly expressed in each tissue of the present study to genes expected to be highly expressed in each tissue based on data from the Allen Brain Atlas (Lein et al., 2007) (Extended Data Figs. 1-1, 1-2, and 1-3).

RNA isolation. RNA isolation was performed according to methods previously described (Ianov et al., 2016; Barter et al., 2019) from subregions of a hippocampus from a single hemisphere of each animal. Briefly, RNA was isolated using the RNeasy Lipid Tissue Mini kit (QIAGEN, catalog \#74804). Total RNA was treated with DNase using the RNase-Free DNase Set (QIAGEN, catalog \#79254). Concentration of total RNA was assessed using the NanoDrop 2000 spectrophotometer (Thermo Fisher Scientific, catalog \#ND-2000), and the RNA integrity number was quantified using the Agilent 2200 Tapestation system with High Sensitivity RNA Screen Tape (Agilent Technologies). Mean RNA integrity number across all samples was 9.05 $(\mathrm{SEM} \pm 0.02)$ with values ranging from 8.2-9.3. Poly-A-selection for mRNA was performed using $250 \mathrm{ng}$ of isolated total RNA in the Dynabeads mRNA DIRECT Micro kit (Thermo Fisher Scientific, catalog \#61021). External RNA Controls Consortium (ERCC) RNA Spike-In Control Mixes were included in all isolated mRNA samples (Thermo Fisher Scientific, catalog \#4456740).

RNA sequencing. Library preparation was performed using the Ion Total RNA-seq Kit version 2 (Thermo Fisher Scientific, catalog \#4475936). Ion Xpress barcodes (Thermo Fisher Scientific, catalog \#4475485) were included with libraries for multiplex sequencing. In summary, isolated mRNA was fragmented with RNase III then ligated to the Ion Adapter Mix version 2. RNA samples were then reverse-transcribed. cDNA from each biological replicate was uniquely barcoded and amplified with 16 PCR cycles. Library concentration from each sample was quantified using the Qubit dsDNA HS Assay (Thermo Fisher Scientific, catalog \#Q32851). DNA fragment size distribution was subsequently evaluated using High Sensitivity D1000 ScreenTape in the 2200 Tapestation system (Agilent Technologies).

Templates were prepared using the Ion Chef system (Thermo Fisher Scientific, catalog \#4484177), and sequencing was performed using the Ion Proton system (Thermo Fisher Scientific, catalog \#4476610) or Ion GeneStudio S5 system (Thermo Fisher Scientific, catalog \#A38194). A principle component analysis performed in the Partek Flow servers (Partek), comparing counts normalized by the trimmed mean of M- values method of all samples run on the Ion Proton System $(n=45)$ with all samples run on the Ion GeneStudio S5 system $(n=39)$, indicated no systematic difference between the two sequencing devices (Extended Data Fig. 1-4). Extended Data Fig. 1-5 lists the platform used to sequence each individual sample. Two-tailed independent samples $t$ tests demonstrated no significant difference between the two sequencing platforms between DI scores $(p=0.79)$ or average equidistant errors $(p=0.13)$. Two-sided Fisher's exact tests demonstrated no significant difference between platforms for age $(p=0.38)$ or tissue type $(p=0.76)$.

RNA sequencing analysis. ERCC analysis was performed with the ERCC analysis plugin in Torrent Suite Software version 5.10.1 (Thermo Fisher Scientific). All spiked samples had an $R^{2}>0.9$. Ion Torrent and Ion GeneStudio-derived reads were then aligned to the rattus norvegicus genome (rn6) using STAR 2.5.3a in the Partek Flow servers (Partek). Gene read counts were generated from Binary Alignment Map files and annotated to the genome using Ensembl (version 92; Partek). Mean number of reads across all samples was (in millions) 32.18 ( \pm 0.37 SEM), ranging from 26.01 to 40.78 . Reads from FASTQ files produced by the Ion Torrent System were trimmed from both ends until all samples' collective average Phred quality score was $>25$. Reads $<25$ bp were also excluded. This yielded an average read length of $144.4( \pm 1.5$ SEM) bp and average count of 31.56 million reads ( \pm 0.38 SEM) per sample.

Gene filtering was performed according to our previously published work (Blalock et al., 2003; Aenlle et al., 2009; Aenlle and Foster, 2010; Zeier et al., 2011; Ianov et al., 2016, 2017; Barter et al., 2019). Briefly, gene lists were filtered to exclude genes with an average count of $\leq 5$ (averaged across all samples included in a comparison). Normalization and pairwise differential expression analysis, including calculation of false discovery rate (FDR), were conducted using the DESeq2 package (version 3.5) for comparisons by age. A statistical filter was then applied with a threshold of significance for DESeq2 pairwise analyses set at $p<0.025$ (age $p$ value). This filtered list of genes was used for gene enrichment clustering analysis for age effects and examining gene clusters for age-related genes that correlated with behavioral measures in middle-age animals (see below).

To assess the relationship of age-related differences in gene expression and cognitive performance, Spearman correlations were calculated between normalized counts of gene expression for middle-age animals within each brain region and either the average number of errors per trial when beginning trials from the equidistant start points across all days of testing or the DI score measured during the probe task. The criterion for a significant correlation, limited to subjects in the middle-age group, was set at $p<0.05$, consistent with our previous work (Blalock et 
al., 2003). An FDR was calculated for genes that passed both age and correlation thresholds. Genes were ranked by the combined $p$ value ( $p$ value age $\times p$ value for behavioral correlation), and the FDR for each rank $\left(q_{\mathrm{i}}\right)$ was calculated by the formula $q_{\mathrm{i}}=\left(p\right.$ value age $\mathrm{e}_{\mathrm{i}} \times p$ value for behavioral correlation $\left._{\mathrm{i}}\right) \times$ total genes examined/i). The Spearman correlation was used, as it has been previously validated for differential correlation analysis in sequencing data (Siska and Kechris, 2017), and there was no assumption within the current study of a normal distribution for either behavioral variable assessed. As genes whose expression is altered with age are of primary interest in this study, only genes that met the significance threshold for both age and correlation were reported. Multiple regression analyses were performed in $\mathrm{R}$ ( $\mathrm{R}$ Core Team, 2019).

Confidence in the significance of individual genes is low due to false positives associated with multiple comparisons across all genes. Gene enrichment analysis was performed under the assumption that changes in biological processes with age or cognition would result in a shift in the expression of clusters of genes related to the biological process (Ianov et al., 2016). Gene enrichment and functional annotation clustering analysis was conducted using the National Institutes of Health database for annotation, visualization, and integrated discovery (DAVID, version 6.8) (Huang et al., 2009) using Gene ontology (GO) for biological process, cellular compartment, and molecular function in the "Direct" and "FAT" categories, in addition to Kyoto Encyclopedia of Genes and Genomes (KEGG) pathways. Benjamini FDR was set at $p<0.05$ as a threshold for cluster selection. For all functional annotation results, we reported significant GO terms among cellular compartment, molecular function, and biological process categories for each annotation cluster. Significant KEGG pathways were also reported. Separate functional cluster analyses were performed for each brain region (DG, CA1, and CA3) for genes filtered for age alone or age and behavioral measures. Analyses were further split by direction of fold change (in the case of age effects) or correlation (in the case of cognitive measures). All counts of filtered genes, including those used in DESeq2 and correlation analyses, include only genes indexed in DAVID. FASTQ files derived from RNA sequencing were submitted to the National Center for Biotechnology Information's Gene Expression Omnibus under accession number GSE140420.

\section{Results}

\section{Behavior}

\section{Cue discrimination training}

Figure 1 illustrates the timeline of behavioral testing. Consistent with previous work (Guidi et al., 2014), repeated-measures ANOVA indicated an effect of training $\left(F_{(4,192)}=17.83\right.$, $\left.p<0.001, \eta^{2}=0.27\right)$ and an effect of age $\left(F_{(1,48)}=23.81\right.$, $\left.p<0.001, \eta^{2}=0.33\right)$ in the absence of an effect of cohort or any interactions (Fig. 2A). Post hoc ANOVAs for each age group indicated that both groups decreased their escape swim distance with training ( $p<0.001$ in each case). All animals were able to locate the escape platform within $1 \mathrm{~min}$ by the end of cue training. The absence of a cohort effect justified further comparisons within cohorts tested at different beacon separations.

\section{Beacon discrimination task}

A repeated-measures ANOVA was conducted for the average number of times animals visited the incorrect beacon per trial (i.e., average errors), with between-subjects factors of age and beacon separation and within-subjects factors of start position and day of training. This analysis indicated main effects of training across days $\left(F_{(2.6,122.4)}=8.00, p<0.0001, \eta^{2}=0.14\right)$, due to decreased errors with training, an effect of age $\left(F_{(1,48)}=24.12\right.$, $\left.p<0.0001, \eta^{2}=0.33\right)$, due to more errors by older animals, and an effect of beacon separation $\left(F_{(1,48)}=47.10, p<0.0001, \eta^{2}=\right.$ 0.50 ), due to increased errors among animals tested at $45 \mathrm{~cm}$ beacon separation. An interaction was observed for days of training $\times$ age $\left(F_{(2.6,122.4)}=3.38, p=0.03, \eta^{2}=0.066\right)$, due mainly to better performance by younger animals on days 3 and 4 (Fig. $2 B$ ). The results indicate that older animals exhibit poorer performance than young, regardless of beacon separation, that both age groups increased accuracy over the course of training, and that discrimination became more difficult as the beacons exhibited less spatial separation.

A main effect was observed for start position $\left(F_{(1.5,71.2)}=\right.$ 51.02, $\left.p<0.0001, \eta^{2}=0.52\right)$, as well as an interaction of start location and beacon separation $\left(F_{(1.5,71.2)}=8.91, p=0.001, \eta^{2}=\right.$ 0.16 ) (Fig. 2C). The number of errors increased as the distance between the escape platform and the start location increased $\mathrm{S}^{-}>$equidistant $>\mathrm{S}^{+}$, indicating that the animals were likely to initially choose the beacon closest to the start location. Finally, post hoc tests for effects of beacon separation at each start position indicated an increase in errors at the $45 \mathrm{~cm}$ separation relative to the $73 \mathrm{~cm}$ separation for trials starting from the $\mathrm{S}^{-}(p<0.001)$ or equidistant point $(p<0.01)$.

Post hoc tests to localize age effects at each separation and start position indicated that middle-age animals made more errors than young when beginning at the equidistant start position, whether tested at $45 \mathrm{~cm}(p<0.01)$ or $73 \mathrm{~cm}(p<0.001)$ separation, and when beginning trials at the $S^{+}$position at $73 \mathrm{~cm}$ separation $(p=0.02)$. Together, the results distinguish the equidistant start location as the most appropriate for detecting effects of age and beacon separation on performance. This start position also provides the most unbiased visual presentation of the two beacons, making data from this position the most ideal for assessing beacon discrimination performance.

Behavioral differences in middle-age animals could reflect nonspecific motivational or sensorimotor deficits. For example, age-related differences in the cue task are largely due to thigmotaxis, related to the initial stress of the water maze, and differences decrease with exposure to the pool (Foster, 2012; Guidi et al., 2015). To determine whether the impairment in beacon discrimination was due to motivation or sensorimotor function, Pearson's correlations were conducted, within each age group for each beacon separation, between the average number of errors made by each animal at the equidistant starting point and the distance to escape for the last block of cue discrimination training. The results did not reveal a relationship between performance on the cue discrimination task and beacon discrimination performance for either age group at either beacon separation (young, $45 \mathrm{~cm}, r=0.16$, $p=0.63$; middle-age, $45 \mathrm{~cm}, r=0.21, p=0.43$; young, $73 \mathrm{~cm}$, $r=0.28, p=0.44$; middle-age, $73 \mathrm{~cm}, r=0.41, p=0.15)$. The results indicate that beacon discrimination performance is not likely due to nonspecific sensorimotor deficits or motivational differences. Finally, for animals trained in the $45 \mathrm{~cm}$ separation task, the number of equidistant errors increased following the shift in the beacons, relative to the last day of training $\left(F_{(1,26)}=11.25, p<0.01\right.$, $\left.\eta^{2}=0.3\right)$ in the absence of an age effect $(p=0.41)$, indicating that both age groups had learned to discriminate the beacon/location (Extended Data Fig. 2-1).

\section{Spatial reference memory assessment}

One day after the last beacon discrimination test, a single probe trial was conducted with the beacons and the platform removed to determine whether animals had formed a spatial reference memory of the platform location. An examination of DI scores for the reference memory probe trial indicated an effect of beacon separation $\left(F_{(1,48)}=15.14, p<0.0005, \eta^{2}=0.24\right)$, with higher DI scores for the larger beacon separation, in the absence of an age effect (Fig. 2D). In order to examine the relationship between beacon 
separation performance and the formation of a spatial reference memory, the DI scores for each age group were correlated with the average number of errors for the equidistant starting location for each beacon separation. The average number of errors for the equidistant starting position in the $45 \mathrm{~cm}$ beacon separation task was positively correlated with the DI score for middle-age animals, such that animals that exhibited more errors discriminating the beacons were more likely to use a reference memory $\left(r^{2}=0.52, p<0.005 ;\right.$ Fig. $\left.2 E\right)$. The distribution of DI scores for young animals also suggests individual variability in the use of an allocentric strategy. However, in contrast to older animals, the selection of an allocentric strategy was not associated with beacon discrimination performance. A similar analysis for the $73 \mathrm{~cm}$ beacon separation did not indicate a relationship between beacon discrimination and spatial reference memory for either age group (data not shown). The results suggest that, for the larger interbeacon distance, animals were more likely to rely on a spatial reference memory strategy. In contrast, for the smaller interbeacon distance, middle-age animals that were impaired for beacon discrimination were more likely to use a spatial reference memory strategy, and young animals performed well on the beacon discrimination task regardless of the strategy used.

\section{Sequencing}

Age-related transcriptomic changes

In order to examine the transcriptional profile associated with age and beacon discrimination performance, young and middle-age animals behaviorally characterized on the $45 \mathrm{~cm}$ task were examined using next-generation sequencing for gene expression in the three regions of the hippocampus. A total of 2593 genes were upregulated and 2821 were downregulated within the DG in middle-age animals compared with young $(p<$ $0.025 ; \mathrm{FDR}<0.063$ ). CA1 and CA3 exhibited relatively fewer differentially expressed genes with age compared with the DG, with CA1 showing 2149 genes upregulated and 2226 downregulated genes $(p<0.025 ; \mathrm{FDR}<0.077)$, and CA3 showing 1952 genes upregulated and 1847 downregulated with age $(p<0.025$; FDR $<0.091)$ (Fig. 3A,B). Extended Data Fig. 4-1 provides a full list of genes showing significant differential expression with age as well as corresponding FDR-adjusted $p$ values derived from DESeq2.

Functional enrichment analysis with DAVID identified a number of genes clusters altered similarly across all hippocampal subregions with age (Fig. 4A-C, Extended Data Fig. 4-2). Upregulated gene clusters in all brain regions included
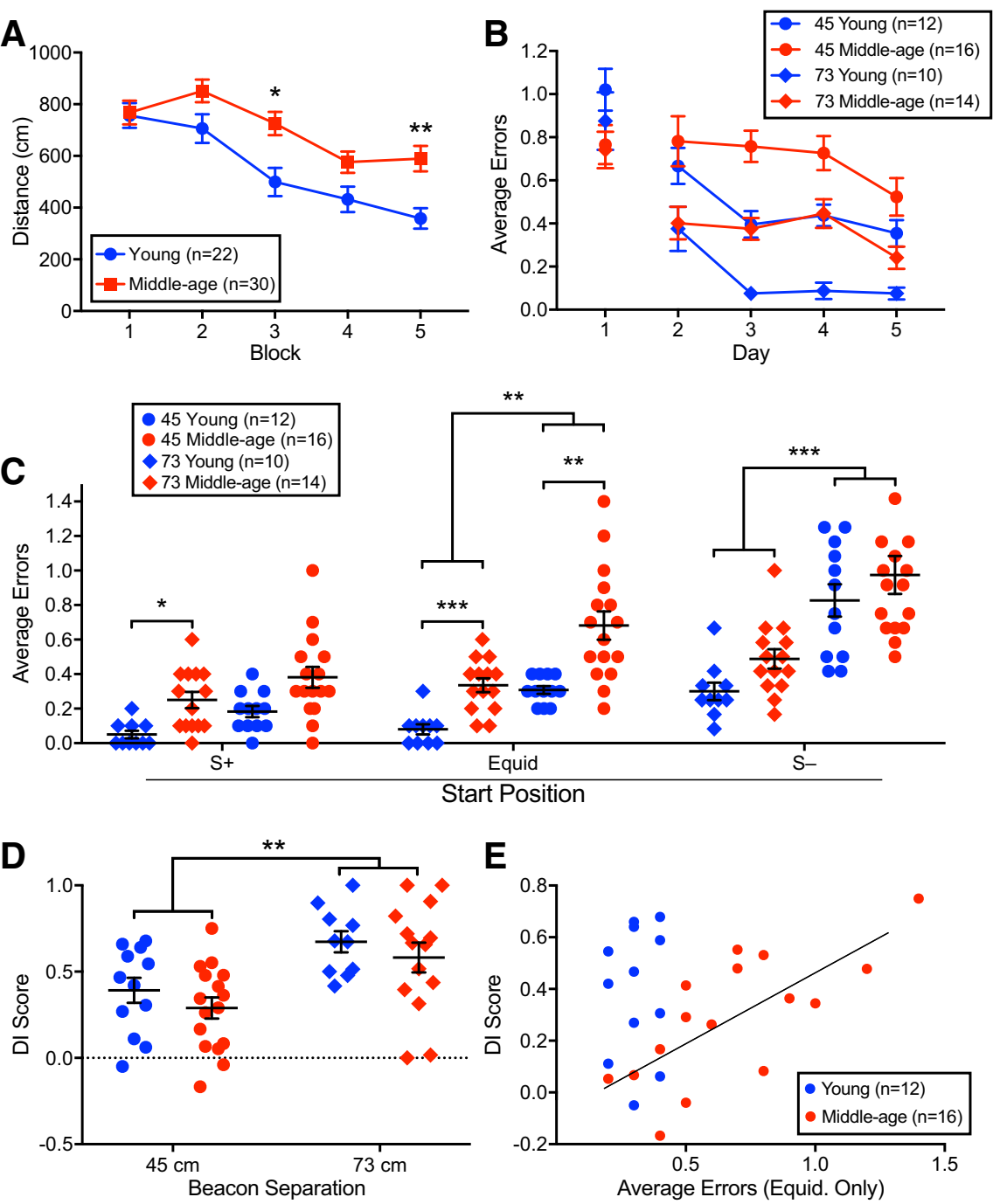

Figure 2. Middle-age animals are impaired relative to young in beacon discrimination, but not spatial reference memory. $\boldsymbol{A}$, Young (blue symbols) and middle-age animals (red symbols) showed improvement in escape swim distance during cue training, despite impaired performance of middle-age animals compared with young. $\boldsymbol{B}$, Average number of errors made per trial (Average Errors) across all days of testing and all trial start positions. Average errors decreased with training, with increased separation (circles represent $45 \mathrm{~cm}$; diamonds represent $73 \mathrm{~cm}$ ), and for young relative to middle-age animals. C, Average errors were influenced by start location such that errors increased as the distance from the start location and goal increased $\left(S^{-}>\right.$equidistant $>S^{+}$) and was higher in animals tested at $45 \mathrm{~cm}$ beacon separation compared with $73 \mathrm{~cm}$ for trials beginning at the equidistant or $S^{-}$start positions. D, DI scores were higher for animals trained at $73 \mathrm{~cm}$ beacon separation compared with $45 \mathrm{~cm}$ separation, and no age difference in DI score was observed for either the 45 or $73 \mathrm{~cm}$ separation group. $\boldsymbol{E}$, Relationship of beacon discrimination performance (average errors from the equidistant starting position for the $45 \mathrm{~cm} \mathrm{sepa-}$ ration) and spatial reference memory (DI score). Within middle-age animals, a significant correlation was noted between DI score and average number of errors made in the beacon discrimination task with trials beginning at the equidistant start point Pearson's $r=0.72, p=0.002$ ). Better reference memory was thus associated with worse beacon discrimination ability. Dotted lines indicate the threshold for chance-level performance. Error bars indicate SEM. ${ }^{*} p<0.05 .{ }^{* *} p<0.01 .{ }^{* * *} p<0.001$.

extracellular exosome (GO:0070062), RNA splicing (GO:0008380), translational initiation (GO:0006413), response to endoplasmic reticulum stress (GO:0034976), cellular component biogenesis (GO:0044085), and chromosome organization (GO:0051276). Clusters of downregulated genes within all brain regions included glutamatergicsynapse (KEGG rno04724), axon part (GO:0033267), postsynapse (GO:0098794), presynapse (GO:0098793), neuron part (GO:0097458), synapse maturation (GO:0060074), cell death (GO:0008219), regulation of catabolic process (GO:0009894), CNS development (GO:0007417), transcription from RNA polymerase II promoter (GO:0006366), and regulation of neuronal synaptic plasticity (GO:0048168). 
A

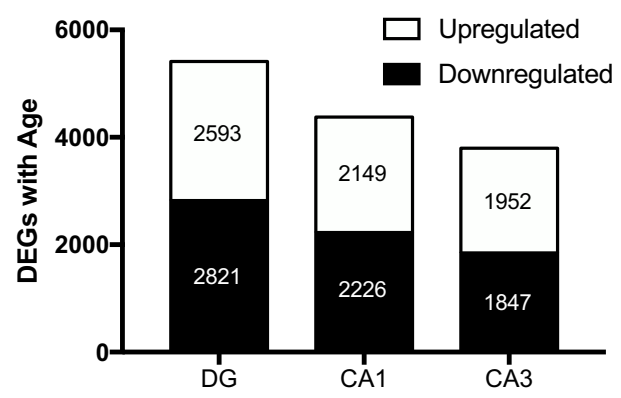

B

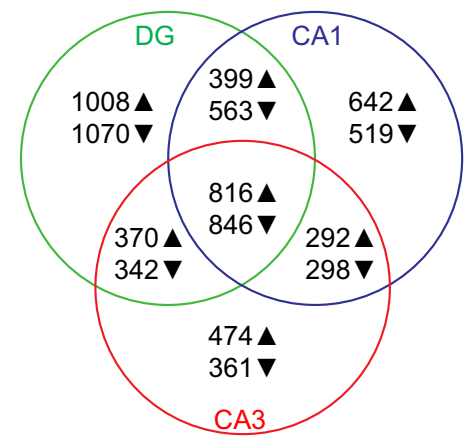

Figure 3. Age is associated with differential regulation of genes within the $D G, C A 1$, and CA3. $A$, By middle-age, the DG was found to show the greatest number of DEGs relative to young rats. $\boldsymbol{B}$, The number of DEGs in common among different brain regions. Arrowheads indicate the direction of differential gene expression. All gene counts represent genes that passed our statistical filter for age and were indexed in DAVID.

Within the DG, clusters with the lowest $p$ values for genes upregulated with age included cellular localization (GO:0051641), RNA metabolic process (GO:0016070), spliceosomal complex (GO:0005681), and centrosome (GO:0005813) (Fig. 4A). The most significant clusters of downregulated genes in the DG included nervous system development (GO:0007399), regulation of signaling (GO:0023051), neuron part (GO:0097458), synapse (GO:0045202), glutamatergic synapse (KEGG rno04724), and protein modification process (GO:0036211) (Fig. 4A, Extended Data Fig. 4-3). These results are consistent with studies describing a reduction in neurogenesis and synapse number in the DG with age. Extended Data Figs. 4-4, 4-5, and 4-6 provide examples of the distribution of a selection of genes from the DG, CA1, and CA3, respectively, which were differentially regulated with age, and associated with significant GO terms.

\section{Cognition-related transcriptomic changes}

As noted above, the average errors for the equidistant start location (equidistant average errors) was the most sensitive and conceptually appropriate for detecting beacon discrimination deficits in middle-age animals. Because of the lack of variability in equidistant average errors for young animals, analysis of the relationship between gene expression and discrimination behavior on the $45 \mathrm{~cm}$ beacon separation task was limited to middleage animals. Spearman's correlations were used to examine the relationship of equidistant average errors and expression of genes that differed across age groups. From the list of DEGs that were different with age in the DG, 329 genes were positively correlated with equidistant errors and 539 genes were negatively correlated with equidistant errors. CA1 expressed 69 and 77 genes positively and negatively correlated with errors, respectively, that were also significantly altered with age. Finally, CA3 showed 51 and 76 genes that were altered with age and exhibited a positive and negative correlation with errors, respectively (Fig. 5A). Cluster enrichment analysis indicated that DG genes, which were differentially expressed with age and negatively correlated with errors (i.e., increasing expression with better performance), included synapse part (GO:0044456), synaptic signaling (GO:0099536), nervous system development (GO:0007399), and neurogenesis (GO:0022008) (Fig. 5B). Unabridged lists of these clusters are provided in Extended Data Fig. 5-1, and a full list of individual genes with significant correlations to errors, along with FDR-adjusted $p$ values is provided in Extended Data Fig. 5-2. Figure 6 provides representative plots of DG synaptic or neurogenesis genes that were significantly different with age and negatively correlated with beacon discrimination errors.

The use of an allocentric strategy, as measured by the probe trial DI scores, exhibited large variability regardless of age and was not different across age groups. To examine the possibility that the DI score was associated with similar gene expression across age groups, we performed multiple regression analysis to control for age. We filtered the genes such that they exhibited an overall significance $(p<0.05)$ for the multiple regression, a significant $(p<0.05)$ correlation with the DI scores, and were not different by age $(p>0.05)$. CA1 exhibited the most genes positively (73) and negatively (74) correlated with the DI score, followed by the DG (46 positively and 53 negatively correlated), then CA3 (38 positively and 35 negatively correlated). Examination of enrichment clustering was null for each region. However, several of the CA1 genes that negatively ( $W d r 5, \operatorname{Med} 20$, Sel1l3, Igfbp6, Thsd7b, Recql5, Olfm2, Atp2b4, Fxyd6) or positively (Ppp3ca, Cyp1b1, Ythdc1, Arhgef37, Cdo1, Tac1, Grm8) correlated with the DI scores have previously been reported to decrease or increase expression, respectively, in CA1 of aged animals with intact spatial reference memory, compared with older memory impaired animals (Rowe et al., 2007; Masser et al., 2014; Ianov et al., 2017).

To determine whether the DI score, a measure of the use of an allocentric strategy, was linked to CA1 genes that change with age, we used the list of genes that were different with age to examine the relationship between gene expression and DI scores in middle-age animals. For CA1, 54 and 77 genes that were different with age exhibited a positive or negative correlation with the DI scores, respectively, in middle-age animals. Gene clustering indicated no enrichment of positively correlated genes, and negatively correlated genes exhibited clusters of neuronal genes, including genes for voltage-gated cation channel activity (GO:0022843). Interestingly, Fos, which has previously been reported to decrease in regions CA1 of animals that preferentially use an allocentric strategy (Fouquet et al., 2013; Yagi et al., 2016), was negatively correlated with DI scores. Figure 7 depicts representative plots of individuals genes altered with age and correlated with DI score within CA1. A full list of genes that were differentially expressed with age and exhibited a significant correlation to DI score within CA1, including FDR-adjusted $p$ values, is provided in Extended Data Fig. 7-1. The results are consistent with previous work indicating that successful formation of a spatial reference memory does not result in the same pattern of gene expression in young and older animals, and suggest that some gene changes represent compensation to maintain function of CA1.

\section{Discussion}

For studies examining multiple hippocampal subregions, little correlation is observed between DG gene expression and hippocampal-dependent spatial memory. Rather, an age-related decline in spatial memory is associated with transcription in regions CA1 and CA3 (Haberman et al., 2011; Masser et al., 2014; Ianov et al., 2017). The lack of DG transcriptional correlates may be due to the use of tasks that do not emphasize cognitive processes that depend on the DG (Ianov et al., 2017). 

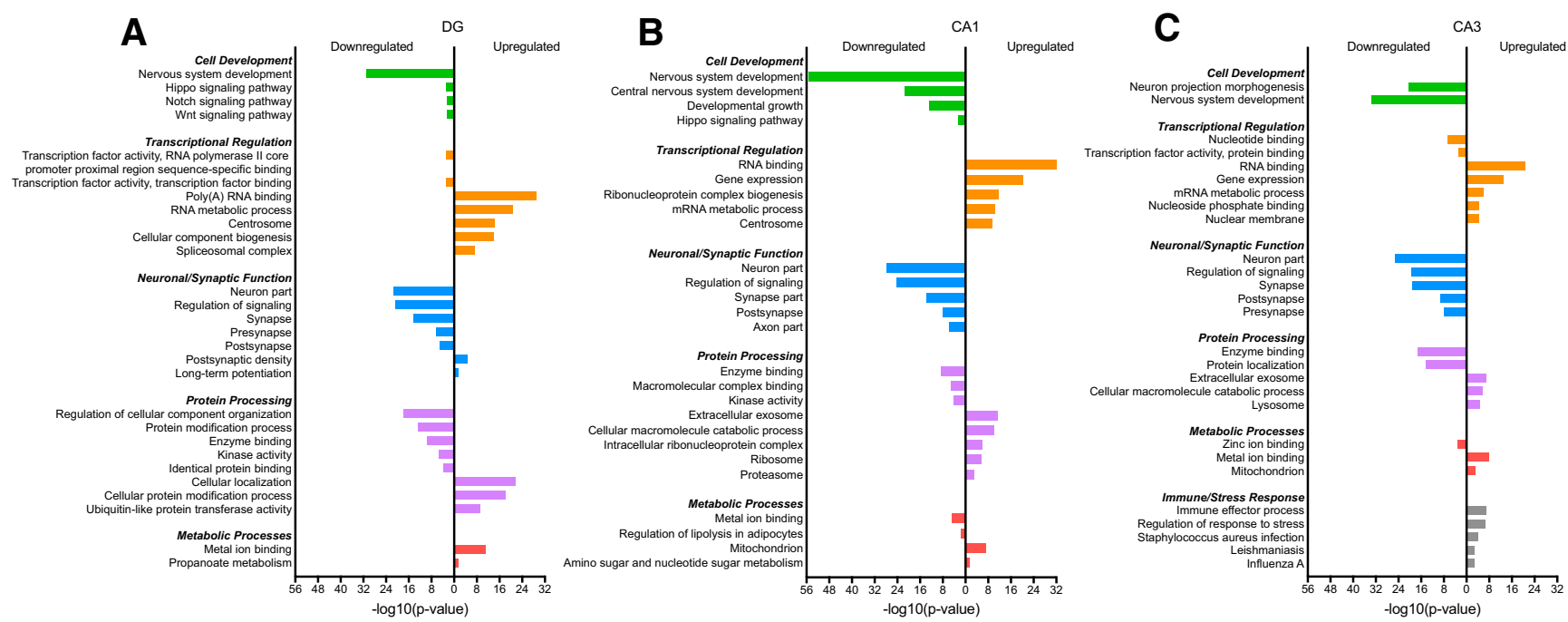

Figure 4. Functional annotation clustering shows substantial differential gene expression by middle-age compared with young animals in the DG $(A), C A 1(B)$, and CA3 (C). Graphs represent the top $G 0$ and KEGG terms of the DEGs of middle-age rats relative to young. The complete list of significant GO and KEGG terms for each tissue is provided in Extended data Figure 4-3. Gene categories showing common differential regulation in all hippocampal subregions with age are provided in Extended data Figure 4-2.

A
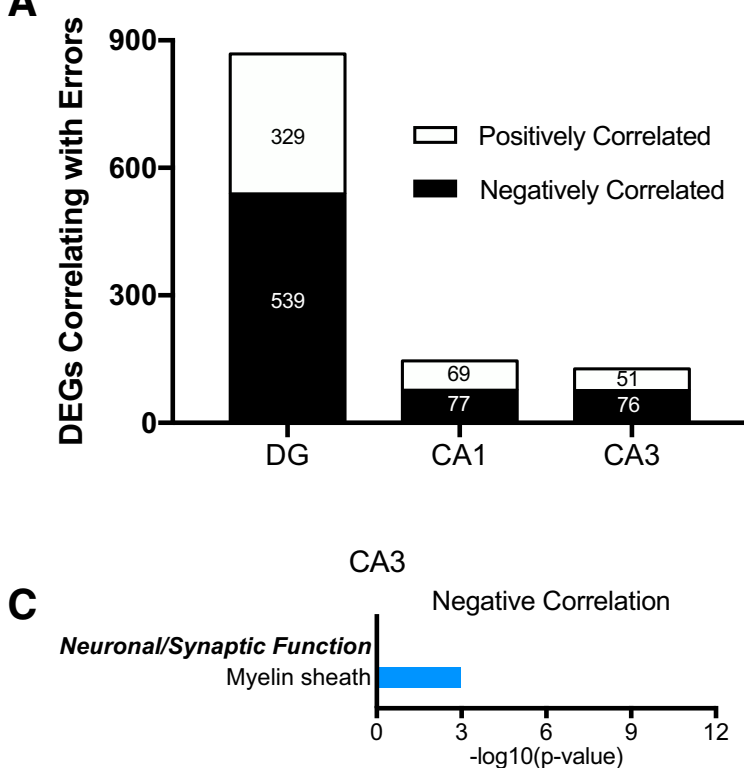

B

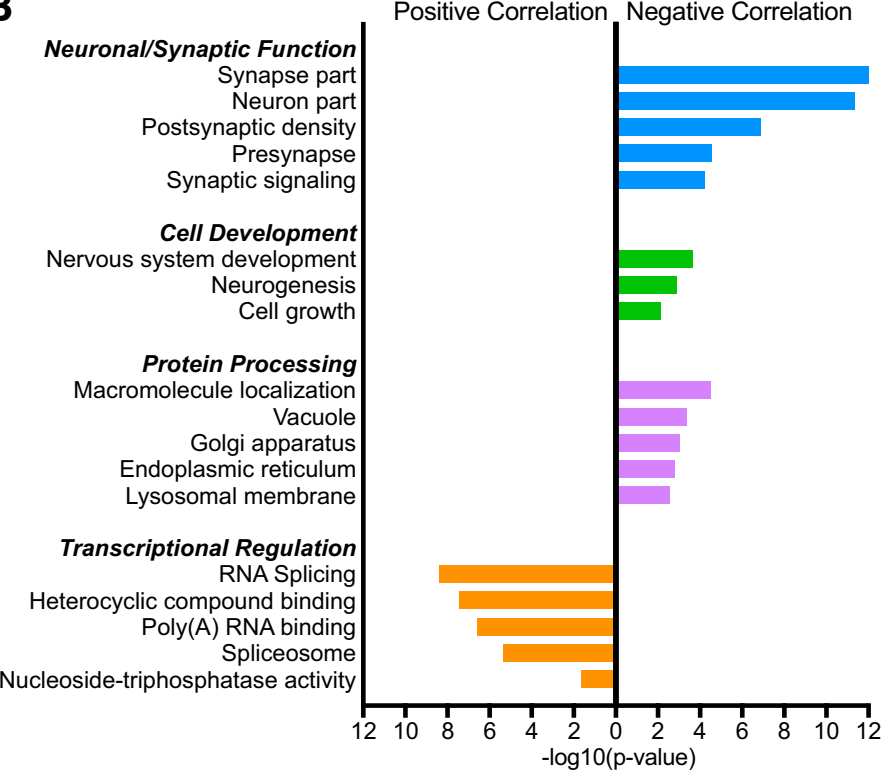

Figure 5. Beacon discrimination performance is correlated with differential regulation of genes primarily within the DG. $A$, Among all hippocampal subregions tested, the DG showed the greatest number of genes correlating with beacon discrimination performance on the $45 \mathrm{~cm}$ beacon separation task within middle-age animals. $\boldsymbol{B}$, The DG showed the greatest number of gene clusters correlating with beacon discrimination performance, including downregulation of genes related to the synapse (G0:0044456) and neurogenesis (G0:0022008) with worse beacon discrimination performance. C, CA3 featured downregulation of genes relating to the myelin sheath (G0:0043209) with worse beacon discrimination performance. All values represent genes, found to pass our statistical filters for age and correlation with beacon discrimination performance within middle-age rats tested at $45 \mathrm{~cm}$ beacon separation only, which were also indexed in DAVID.

Impaired DG function is thought to contribute to an agerelated decline in the ability to perform pattern separation tasks, observed as an impairment in the ability to distinguish objects as feature overlap increases or discriminate between the locations of two identical stimuli as the distance between objects is diminished, in humans (Bakker et al., 2008; Yassa et al., 2011; Holden et al., 2012; Reagh et al., 2018) and animal models (Creer et al., 2010; Burke et al., 2011; Gracian et al., 2013; Gray and Barnes, 2015; Wu et al., 2015; Johnson et al., 2016, 2017; Cès et al., 2018).
Similar to aging, DG lesions, decreased neurogenesis, or disruption of DG synaptic function is associated with impairment on pattern separation tasks (Gilbert et al., 2001; McHugh et al., 2007; Hunsaker et al., 2008; Clelland et al., 2009; Bannerman et al., 2012; Morris et al., 2012; Taylor et al., 2013). In contrast, the role of the DG and neurogenesis in spatial reference memory is more nuanced (Xavier et al., 1999; Bizon et al., 2004; McHugh et al., 2007; Epp and Galea, 2009; Bannerman et al., 2012; Morris et al., 2012; Taylor et al., 2013; Barha et al., 2015; Coradazzi et al., 2016). 

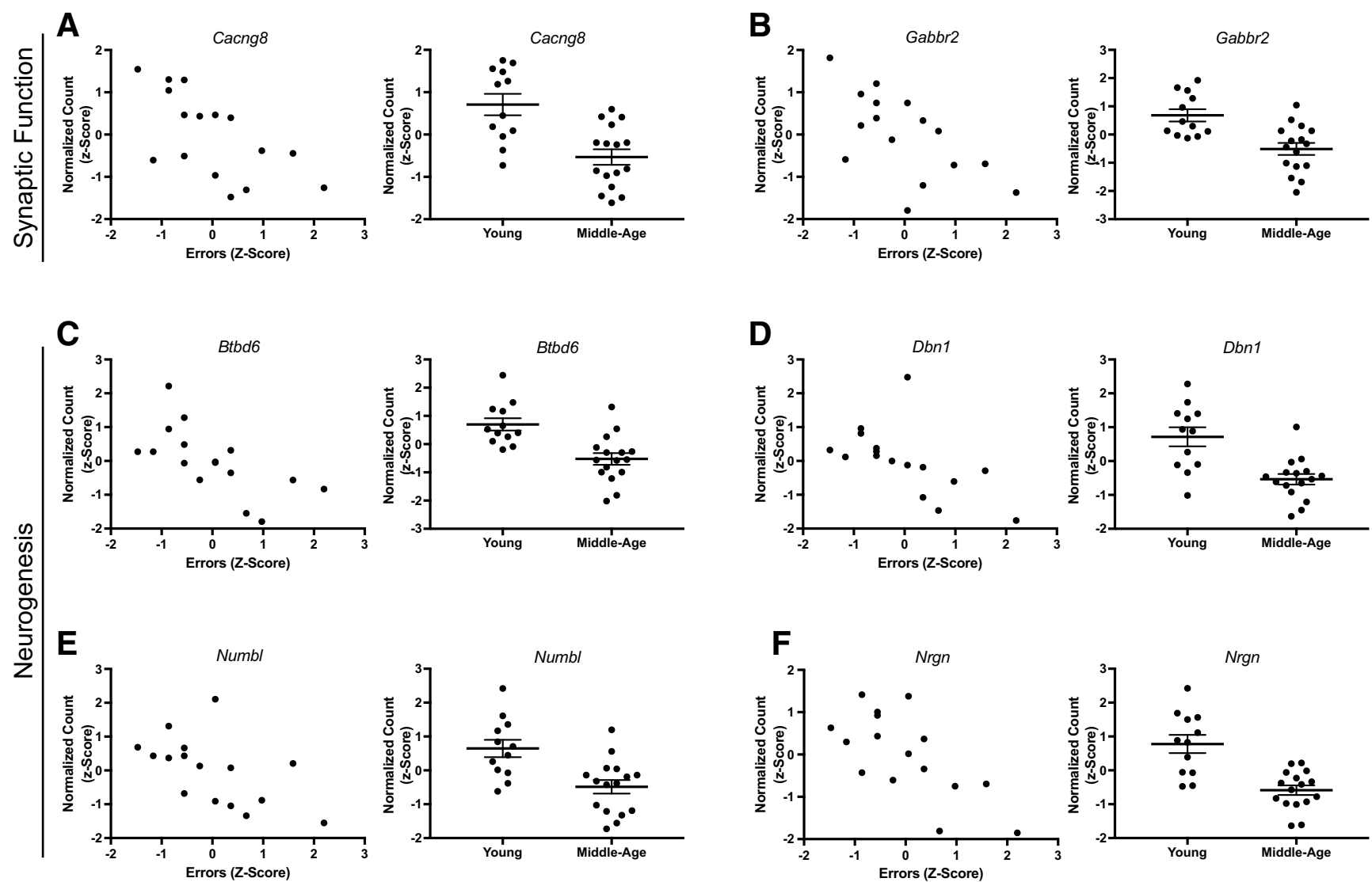

Figure 6. Examples of DG genes that are differentially expressed with age and significantly correlated with equidistant average errors in middle-age animals. Each gene depicted shows decreased expression in animals with worse beacon discrimination performance. For plots of age differences, data in the $y$ axes reflect the $z$ distributions of normalized gene counts within both young and middle-age animals. For plots depicting correlations for middle-age animals, the $y$ axes reflect the $z$ distributions of normalized gene counts for middle-age animals only. In correlation figures, the $x$ axes represent the $z$ distributions of average equidistant errors within middle-age animals only. Each gene depicted shows decreased expression in animals with worse beacon discrimination performance. $\boldsymbol{A}, \boldsymbol{B}$, Genes from the synapse part (G0:0044456). $\mathbf{C}-\boldsymbol{F}$, Genes associated with neurogenesis (G0:0022008).

Animals can use a variety of cognitive strategies, associated with different brain networks, to solve tasks. Moreover, different cognitive strategies and associated brain networks may be used to compensate for deficits associated with senescence or pathology of a particular network (Steffener and Stern, 2012). Animals with impaired ability to distinguish objects as feature overlap increases or discriminate between the locations of two identical stimuli as the distance between objects is diminished can use cue-response or allocentric strategies to locate goals (Costa et al., 2005; Morris et al., 2012; Bannerman et al., 2014). For example, aged or lesioned animals, impaired in the ability to distinguish similar objects, shift egocentric strategies and exhibit response bias as feature overlap increases (Snigdha et al., 2017; Burke et al., 2018). In addition, animals can use an allocentric strategy to compensate for impaired ability to discriminate between adjacent locations (Gilbert et al., 2001; Morris et al., 2012; Bannerman et al., 2014). For $\mathrm{S}^{+}$and $\mathrm{S}^{-}$start positions, animals typically choose the closest beacon, indicating an egocentric or cue-response strategy. For the equidistant start locations, errors increased as the distance between the cues decreased, and errors increased with age, confirming that deficits emerge by middle age (Huxter et al., 2012; Stark et al., 2013). Probe trials indicated that, when the beacons were far apart, animals exhibited an allocentric strategy and formation of a spatial reference memory. For the $45 \mathrm{~cm}$ beacon separation, probe trial data indicated that middle-age animals with increased errors were more likely to use an allocentric strategy, concentrating their search on the spatial location. In contrast, animals with fewer equidistant errors exhibited search behavior that was not focused on the spatial location, indicating that they relied on cue-response behavior to discriminate the beacons.

Differences in gene expression suggest that impaired beacon discrimination results from senescence within the task-related network, specifically the DG. Although most studies focus on young and aged animals, previous research indicates large changes in expression in the hippocampus and in the DG between young adult and middle-age (Blalock et al., 2003; Ianov et al., 2017). Furthermore, the DG exhibits several neurobiological changes by middle-age (Dieguez and Barea-Rodriguez, 2004; Twarkowski and Manahan-Vaughan, 2016; McGuiness et al., 2017). In particular, neurogenesis declines in middle-age (Seki and Arai, 1995; Lichtenwalner et al., 2001; Bizon and Gallagher, 2003; Bondolfi et al., 2004; Driscoll et al., 2006; Kronenberg et al., 2006; McGuiness et al., 2017). The current study supports the idea that the number of genes correlated with behavior is increased by examining brain networks that contribute to the specific behavior, including medial prefrontal cortex genes and impaired executive function (Ianov et al., 2016), and region CA1 genes and impaired spatial memory (Masser et al., 2014; Ianov et al., 2017). Thus, the gene differences likely represent neurobiological processes that are changing with age and contribute to impaired function of the circuit.

In agreement with previous studies (Burger et al., 2008; Masser et al., 2014; Ianov et al., 2016), we observed upregulation 

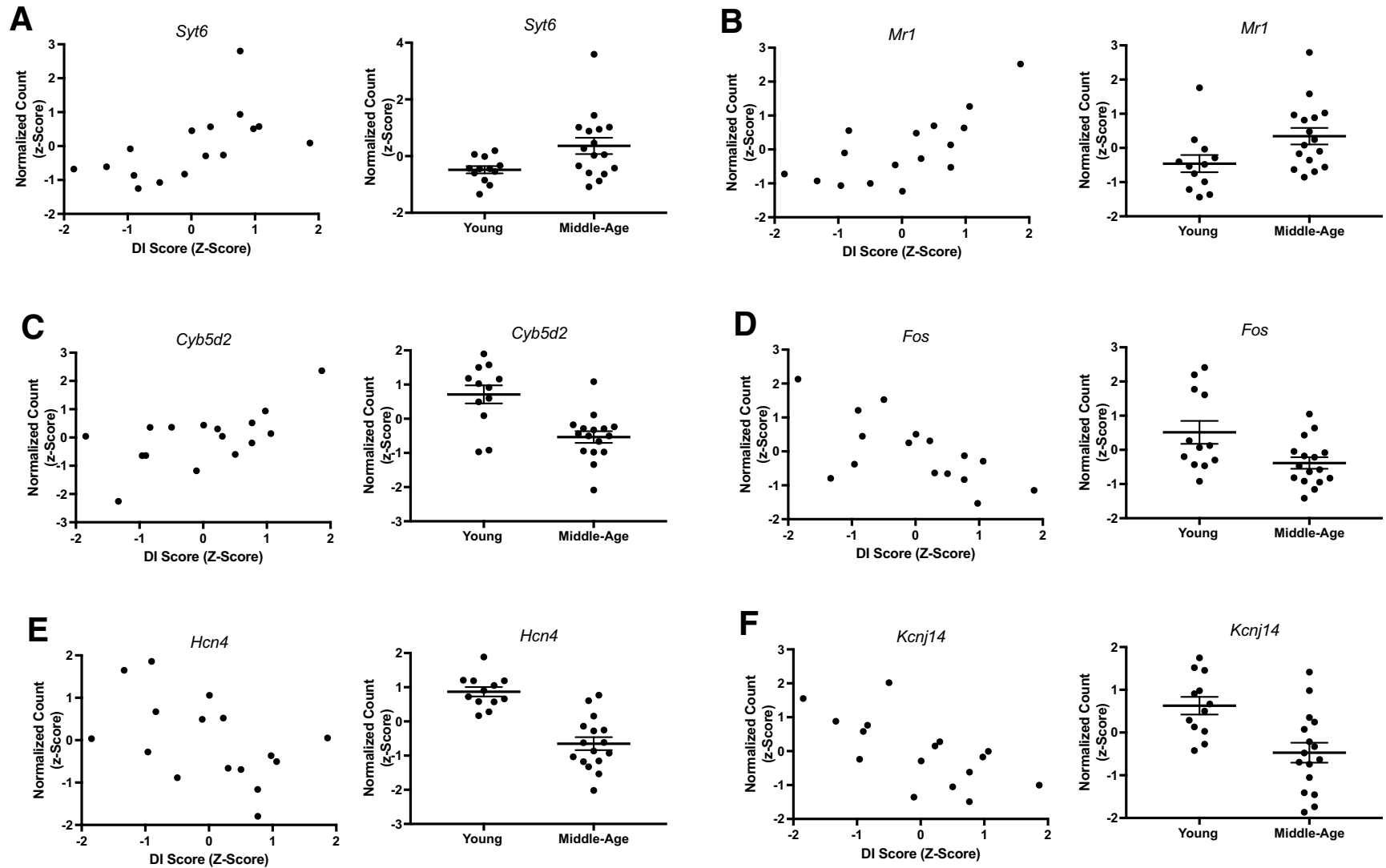

Figure 7. Examples of CA1 genes that are differentially expressed with age and significantly correlated with DI score in middle-age animals. For plots of age differences, data in the $y$ axes reflect the $z$ distributions of normalized gene counts within both young and middle-age animals. For plots depicting correlations for middle-age animals, the $y$ axes reflect the $z$ distributions of normalized gene counts for middle-age animals only. In correlation figures, the $x$ axes represent the $z$ distributions of DI scores within middle-age animals only. Animals with better reference memory performance, reflected by greater DI score, showed increased expression of Syt6 (A), Mr1 (B), and Cyb5d2 (C); and decreased expression of Fos (D), Hcn4 (E), and Kcnj14 (F). A full list of genes showing a significant correlation to DI score is provided in Extended data Figure 7-1.

of immune (Apobec1, B2m, Cd4b, Cd74, Fcerlg, Fcgr2b, Lrrc17, $L y v, M s h 2, R T 1-D a, R T 1-D b 1, R T 1-B a$, Serpinbp, Syk) and lysosomal genes (Cd74, Ctss, Myo5a, Pon2, Rab14, RT1-Da, RT1$D b 1, R T 1-B a)$ in the DG of older animals. Importantly, immune response genes did not correlate with beacon discrimination performance. While cognitive impairment may be related to physiological changes associated with neuroinflammation, the results are consistent with the observation that immune response genes do not predict cognitive impairment (Haberman et al., 2011; Masser et al., 2014; Ianov et al., 2017).

Beacon discrimination impairment was associated with a decrease in DG synaptic genes, consistent with age-related changes in DG afferents and synaptic plasticity (Gray and Barnes, 2015) and studies indicating that impaired DG synptic function contributes to impaired pattern separation behavior (McHugh et al., 2007; Bannerman et al., 2014; Kannangara et al., 2015). The decrease in synaptic genes is also consistent with decreased neurogenesis (Chatzi et al., 2016). Aging is characterized by delayed maturation of new DG neurons and a decline in total new neurons (Bizon et al., 2004; Speisman et al., 2013; McGuiness et al., 2017; Toda and Gage, 2018). In turn, decreased neurogenesis has been linked to impaired pattern separation behavior (Clelland et al., 2009; Aimone et al., 2011; Sahay et al., 2011; Toda and Gage, 2018).

The role of neurogenesis in spatial memory is unclear (Shors et al., 2002), with several studies indicating no relationship, or decreased neurogenesis associated with increased use of an allocentric strategy (Bizon et al., 2004; Epp and Galea, 2009; Barha et al., 2015; Coradazzi et al., 2016). For the $45 \mathrm{~cm}$ beacon separation, both age groups exibited similar variability in the DI score for the probe trial. When both age groups were examined together, few genes were correlated with the DI score, consistent with work indicating that spatial memory behavior does not result in the same pattern of gene expression in young and older animals (Burger, 2010). Interestingly, within a multiple regression model controlling for age, CA1 exhibited the most genes whose expression correlated to DI score, including several that have previously been reported to differentiate age-impaired and aged-unimpaired for spatial memory, including genes for regulating neural activity. These cognition-related genes may represent an attempt by CA1 neurons to compensate for senescent changes (Ianov et al., 2017; Foster, 2019). Interestingly, Fos was negatively correlated with DI scores for middle-age animals (i.e., decreased expression in animals that used an allocentric strategy). The results support work demonstrating reduced c-fos expression in region CA1 of animals that preferentially use an allocentric strategy (Fouquet et al., 2013; Yagi et al., 2016). Finally, it is likely that genes and gene clusters were missed due to the stringency of analysis. Other studies have observed differences when comparing age-impaired or age-unimpaired with each other, regardless of whether the genes were different with age (Haberman et al., 2011; Masser et al., 2014; Ianov et al., 2017).

Together, these results indicate the beacon task is sensitive to age-related cognitive impairment as early as middle-age. Different response strategies used to solve the task were related 
to distinct profiles of gene expression in different hippocampal subfields. The use of different strategies and associated transcriptional profiles may represent examples of cognitive reserve and neural compensation (Stern et al., 2019). Cognitive reserve has been described as an ability to flexibly and efficiently use the best strategies to perform a task, particularly in the face of brain aging or pathology. The variability in beacon discrimination errors associated with strategy selection aligns with human studies that indicate impairment in a specific cognitive domain limits the strategy repertoire, contributing to variance in performance (Hodzik and Lemaire, 2011; Barulli et al., 2013). In turn, impairment within a cognitive domain may result from pathology or senescence within the task-related network. Imaging studies in humans implicate the DG/CA3 network in the age-related decline in object pattern separation (Reagh et al., 2018). The current study indicates that disruption of the network for efficient beacon discrimination involves altered molecular signals for neurogenesis and synaptic function in the DG, and the shift to an allocentric strategy may involve neural compensation in other hippocampal regions. Future studies should examine gene expression in relation to treatments that regulate neurogenesis and synaptic plasticity in the DG and the ability to distinguish objects as feature overlap increases or discriminate between the locations of two adjacent identical stimuli (Bekinschtein et al., 2011).

\section{References}

Aenlle KK, Foster TC (2010) Aging alters the expression of genes for neuroprotection and synaptic function following acute estradiol treatment. Hippocampus 20:1047-1060.

Aenlle KK, Kumar A, Cui L, Jackson TC, Foster TC (2009) Estrogen effects on cognition and hippocampal transcription in middle-aged mice. Neurobiol Aging 30:932-945.

Aimone JB, Deng W, Gage FH (2011) Resolving new memories: a critical look at the dentate gyrus, adult neurogenesis, and pattern separation. Neuron 70:589-596.

Baker S, Vieweg P, Gao F, Gilboa A, Wolbers T, Black SE, Rosenbaum RS (2016) The human dentate gyrus plays a necessary role in discriminating new memories. Curr Biol 26:2629-2634.

Bakker A, Kirwan CB, Miller M, Stark CE (2008) Pattern separation in the human hippocampal CA3 and dentate gyrus. Science 319:16401642.

Bakker A, Krauss GL, Albert MS, Speck CL, Jones LR, Stark CE, Yassa MA, Bassett SS, Shelton AL, Gallagher M (2012) Reduction of hippocampal hyperactivity improves cognition in amnestic mild cognitive impairment. Neuron 74:467-474.

Bannerman DM, Sprengel R, Sanderson DJ, McHugh SB, Rawlins JN, Monyer H, Seeburg PH (2014) Hippocampal synaptic plasticity, spatial memory and anxiety. Nat Rev Neurosci 15:181-192.

Bannerman DM, Bus T, Taylor A, Sanderson DJ, Schwarz I, Jensen V, Hvalby $\varnothing$, Rawlins JN, Seeburg PH, Sprengel R (2012) Dissecting spatial knowledge from spatial choice by hippocampal NMDA receptor deletion. Nat Neurosci 15:1153-1159.

Barha CK, Lieblich SE, Chow C, Galea LA (2015) Multiparity-induced enhancement of hippocampal neurogenesis and spatial memory depends on ovarian hormone status in middle age. Neurobiol Aging 36:23912405.

Barter J, Kumar A, Stortz JA, Hollen M, Nacionales D, Efron PA, Moldawer LL, Foster TC (2019) Age and sex influence the hippocampal response and recovery following sepsis. Mol Neurobiol 56:8557-8572.

Barulli DJ, Rakitin BC, Lemaire P, Stern Y (2013) The influence of cognitive reserve on strategy selection in normal aging. J Int Neuropsychol Soc 19:841-844

Bekinschtein P, Oomen CA, Saksida LM, Bussey TJ (2011) Effects of environmental enrichment and voluntary exercise on neurogenesis, learning and memory, and pattern separation: BDNF as a critical variable? Semin Cell Dev Biol 22:536-542.
Bizon JL, Gallagher M (2003) Production of new cells in the rat dentate gyrus over the lifespan: relation to cognitive decline. Eur J Neurosci 18:215219.

Bizon JL, Lee HJ, Gallagher M (2004) Neurogenesis in a rat model of agerelated cognitive decline. Aging Cell 3:227-234.

Blalock EM, Chen KC, Sharrow K, Herman JP, Porter NM, Foster TC, Landfield PW (2003) Gene microarrays in hippocampal aging: statistical profiling identifies novel processes correlated with cognitive impairment. J Neurosci 23:3807-3819.

Bondolfi L, Ermini F, Long JM, Ingram DK, Jucker M (2004) Impact of age and caloric restriction on neurogenesis in the dentate gyrus of C57BL/6 mice. Neurobiol Aging 25:333-340.

Burger C (2010) Region-specific genetic alterations in the aging hippocampus: implications for cognitive aging. Front Aging Neurosci 2:140.

Burger C, Lopez MC, Baker HV, Mandel RJ, Muzyczka N (2008) Genomewide analysis of aging and learning-related genes in the hippocampal dentate gyrus. Neurobiol Learn Mem 89:379-396.

Burke SN, Wallace JL, Nematollahi S, Uprety AR, Barnes CA (2010) Pattern separation deficits may contribute to age-associated recognition impairments. Behav Neurosci 124:559-573.

Burke SN, Wallace JL, Hartzell AL, Nematollahi S, Plange K, Barnes CA (2011) Age-associated deficits in pattern separation functions of the perirhinal cortex: a cross-species consensus. Behav Neurosci 125:836-847.

Burke SN, Turner SM, Desrosiers CL, Johnson SA, Maurer AP (2018) Perforant path fiber loss results in mnemonic discrimination task deficits in young rats. Front Syst Neurosci 12:61.

Chatzi C, Zhang Y, Shen R, Westbrook GL, Goodman RH (2016) Transcriptional profiling of newly generated dentate granule cells using $\mathrm{TU}$ tagging reveals pattern shifts in gene expression during circuit integration. eNeuro 3:ENEURO.0024-16.2016.

Clark R, Tahan AC, Watson PD, Severson J, Cohen NJ, Voss M (2017) Aging affects spatial reconstruction more than spatial pattern separation performance even after extended practice. Hippocampus 27:716-725.

Clelland CD, Choi M, Romberg C, Clemenson GD, Fragniere A, Tyers P, Jessberger S, Saksida LM, Barker RA, Gage FH, Bussey TJ (2009) A functional role for adult hippocampal neurogenesis in spatial pattern separation. Science 325:210-213.

Coradazzi M, Gulino R, Fieramosca F, Falzacappa LV, Riggi M, Leanza G (2016) Selective noradrenaline depletion impairs working memory and hippocampal neurogenesis. Neurobiol Aging 48:93-102.

Costa VC, Bueno JL, Xavier GF (2005) Dentate gyrus-selective colchicine lesion and performance in temporal and spatial tasks. Behav Brain Res 160:286-303.

Creer DJ, Romberg C, Saksida LM, van Praag H, Bussey TJ (2010) Running enhances spatial pattern separation in mice. Proc Natl Acad Sci USA 107:2367-2372.

Crispino M, Stone DJ, Wei M, Anderson CP, Tocco G, Finch CE, Baudry M (1999) Variations of synaptotagmin I, synaptotagmin IV, and synaptophysin mRNA levels in rat hippocampus during the estrous cycle. Exp Neurol 159:574-583.

Cès A, Burg T, Herbeaux K, Héraud C, Bott JB, Mensah-Nyagan AG, Mathis C (2018) Age-related vulnerability of pattern separation in C57BL/6J mice. Neurobiol Aging 62:120-129.

DiCarlo LM, Vied C, Nowakowski RS (2017) The stability of the transcriptome during the estrous cycle in four regions of the mouse brain. J Comp Neurol 525:3360-3387.

Dieguez D, Barea-Rodriguez EJ (2004) Aging impairs the late phase of longterm potentiation at the medial perforant path-CA3 synapse in awake rats. Synapse 52:53-61.

Driscoll I, Howard SR, Stone JC, Monfils MH, Tomanek B, Brooks WM, Sutherland RJ (2006) The aging hippocampus: a multi-level analysis in the rat. Neuroscience 139:1173-1185.

Epp JR, Galea LA (2009) Hippocampus-dependent strategy choice predicts low levels of cell proliferation in the dentate gyrus. Neurobiol Learn Mem 91:437-446.

Foster TC (2005) Interaction of rapid signal transduction cascades and gene expression in mediating estrogen effects on memory over the life span. Front Neuroendocrinol 26:51-64.

Foster TC (2012) Challenges and opportunities in characterizing cognitive aging across species. Front Aging Neurosci 4:33.

Foster TC (2019) Senescent neurophysiology: $\mathrm{Ca}^{2+}$ signaling from the membrane to the nucleus. Neurobiol Learn Mem 164:107064. 
Fouquet C, Babayan BM, Watilliaux A, Bontempi B, Tobin C, Rondi-Reig L (2013) Complementary roles of the hippocampus and the dorsomedial striatum during spatial and sequence-based navigation behavior. PLoS One 8:e67232.

Gilbert PE, Kesner RP, Lee I (2001) Dissociating hippocampal subregions: a double dissociation between dentate gyrus and CA1. Hippocampus 11:626-636.

Gracian EI, Shelley LE, Morris AM, Gilbert PE (2013) Age-related changes in place learning for adjacent and separate locations. Neurobiol Aging 34:2304-2309.

Gray DT, Barnes CA (2015) Distinguishing adaptive plasticity from vulnerability in the aging hippocampus. Neuroscience 309:17-28.

Guidi M, Kumar A, Rani A, Foster TC (2014) Assessing the emergence and reliability of cognitive decline over the life span in Fisher 344 rats using the spatial water maze. Front Aging Neurosci 6:2.

Guidi M, Rani A, Karic S, Severance B, Kumar A, Foster TC (2015) Contribution of N-methyl-D-aspartate receptors to attention and episodic spatial memory during senescence. Neurobiol Learn Mem 125: $36-46$.

Haberman RP, Colantuoni C, Stocker AM, Schmidt AC, Pedersen JT, Gallagher M (2011) Prominent hippocampal CA3 gene expression profile in neurocognitive aging. Neurobiol Aging 32:1678-1692.

Hodzik S, Lemaire P (2011) Inhibition and shifting capacities mediate adults' age-related differences in strategy selection and repertoire. Acta Psychol (Amst) 137:335-344.

Holden HM, Hoebel C, Loftis K, Gilbert PE (2012) Spatial pattern separation in cognitively normal young and older adults. Hippocampus 22:18261832.

Huang DW, Sherman BT, Lempicki RA (2009) Systematic and integrative analysis of large gene lists using DAVID bioinformatics resources. Nat Protoc 4:44-57.

Huffman DJ, Stark CE (2017) Age-related impairment on a forced-choice version of the Mnemonic Similarity Task. Behav Neurosci 131:55-67.

Hunsaker MR, Rosenberg JS, Kesner RP (2008) The role of the dentate gyrus, $\mathrm{CA} 3 \mathrm{a}, \mathrm{b}$, and $\mathrm{CA} 3 \mathrm{c}$ for detecting spatial and environmental novelty. Hippocampus 18:1064-1073.

Huxter JR, Miranda JA, Dias R (2012) The hippocampal physiology of approaching middle-age: early indicators of change. Hippocampus 22:1923-1940

Ianov L, Rani A, Beas BS, Kumar A, Foster TC (2016) Transcription profile of aging and cognition-related genes in the medial prefrontal cortex. Front Aging Neurosci 8:113.

Ianov L, De Both M, Chawla MK, Rani A, Kennedy AJ, Piras I, Day JJ, Siniard A, Kumar A, Sweatt JD, Barnes CA, Huentelman MJ, Foster TC (2017) Hippocampal transcriptomic profiles: subfield vulnerability to age and cognitive impairment. Front Aging Neurosci 9:383.

Johnson SA, Sacks PK, Turner SM, Gaynor LS, Ormerod BK, Maurer AP, Bizon JL, Burke SN (2016) Discrimination performance in aging is vulnerable to interference and dissociable from spatial memory. Learn Mem 23:339-348.

Johnson SA, Turner SM, Santacroce LA, Carty KN, Shafiq L, Bizon JL, Maurer AP, Burke SN (2017) Rodent age-related impairments in discriminating perceptually similar objects parallel those observed in humans. Hippocampus 27:759-776.

Kannangara TS, Eadie BD, Bostrom CA, Morch K, Brocardo PS, Christie BR (2015) GluN2 $\mathrm{A}^{-/-}$mice lack bidirectional synaptic plasticity in the dentate gyrus and perform poorly on spatial pattern separation tasks. Cereb Cortex 25:2102-2113.

Kronenberg G, Bick-Sander A, Bunk E, Wolf C, Ehninger D, Kempermann G (2006) Physical exercise prevents age-related decline in precursor cell activity in the mouse dentate gyrus. Neurobiol Aging 27:15051513.

Lein ES, Zhao X, Gage FH (2004) Defining a molecular atlas of the hippocampus using DNA microarrays and high-throughput in situ hybridization. J Neurosci 24:3879-3889.

Lein ES, Hawrylycz MJ, Ao N, Ayres M, Bensinger A, Bernard A, Boe AF, Boguski MS, Brockway KS, Byrnes EJ, Chen L, Chen L, Chen TM, Chin MC, Chong J, Crook BE, Czaplinska A, Dang CN, Datta S, Dee NR, et al. (2007) Genome-wide atlas of gene expression in the adult mouse brain. Nature 445:168-176.

Lichtenwalner RJ, Forbes ME, Bennett SA, Lynch CD, Sonntag WE, Riddle DR (2001) Intracerebroventricular infusion of insulin-like growth factor-
I ameliorates the age-related decline in hippocampal neurogenesis. Neuroscience 107:603-613.

Ly M, Murray E, Yassa MA (2013) Perceptual versus conceptual interference and pattern separation of verbal stimuli in young and older adults. Hippocampus 23:425-430.

Masser DR, Bixler GV, Brucklacher RM, Yan H, Giles CB, Wren JD, Sonntag WE, Freeman WM (2014) Hippocampal subregions exhibit both distinct and shared transcriptomic responses to aging and nonneurodegenerative cognitive decline. J Gerontol A Biol Sci Med Sci 69:1311-1324.

McGuiness JA, Scheinert RB, Asokan A, Stadler VC, Lee CS, Rani A, Kumar A, Foster TC, Ormerod BK (2017) Indomethacin increases neurogenesis across age groups and improves delayed probe trial difference scores in middle-aged rats. Front Aging Neurosci 9:280.

McHugh TJ, Jones MW, Quinn JJ, Balthasar N, Coppari R, Elmquist JK, Lowell BB, Fanselow MS, Wilson MA, Tonegawa S (2007) Dentate gyrus NMDA receptors mediate rapid pattern separation in the hippocampal network. Science 317:94-99.

Morris AM, Churchwell JC, Kesner RP, Gilbert PE (2012) Selective lesions of the dentate gyrus produce disruptions in place learning for adjacent spatial locations. Neurobiol Learn Mem 97:326-331.

Prolla TA (2002) DNA microarray analysis of the aging brain. Chem Senses 27:299-306.

R Core Team (2019) R: a language and environment for statistical computing. In. Vienna: R Foundation for Statistical Computing.

Reagh ZM, Roberts JM, Ly M, DiProspero N, Murray E, Yassa MA (2014) Spatial discrimination deficits as a function of mnemonic interference in aged adults with and without memory impairment: spatial discrimination and interference in aging. Hippocampus 24:303-314.

Reagh ZM, Noche JA, Tustison NJ, Delisle D, Murray EA, Yassa MA (2018) Functional imbalance of anterolateral entorhinal cortex and hippocampal dentate/CA3 underlies age-related object pattern separation deficits. Neuron 97:1187-1198.e1184.

Rowe WB, Blalock EM, Chen KC, Kadish I, Wang D, Barrett JE, Thibault O, Porter NM, Rose GM, Landfield PW (2007) Hippocampal expression analyses reveal selective association of immediate-early, neuroenergetic, and myelinogenic pathways with cognitive impairment in aged rats. J Neurosci 27:3098-3110.

Sahay A, Scobie KN, Hill AS, O'Carroll CM, Kheirbek MA, Burghardt NS, Fenton AA, Dranovsky A, Hen R (2011) Increasing adult hippocampal neurogenesis is sufficient to improve pattern separation. Nature 472:466470.

Seki T, Arai Y (1995) Age-related production of new granule cells in the adult dentate gyrus. Neuroreport 6:2479-2482.

Shors TJ, Townsend DA, Zhao M, Kozorovitskiy Y, Gould E (2002) Neurogenesis may relate to some but not all types of hippocampal-dependent learning. Hippocampus 12:578-584.

Siska C, Kechris K (2017) Differential correlation for sequencing data. BMC Res Notes 10:54

Snigdha S, Yassa MA, deRivera C, Milgram NW, Cotman CW (2017) Pattern separation and goal-directed behavior in the aged canine. Learn Mem 24:123-131.

Speisman RB, Kumar A, Rani A, Foster TC, Ormerod BK (2013) Daily exercise improves memory, stimulates hippocampal neurogenesis and modulates immune and neuroimmune cytokines in aging rats. Brain Behav Immun 28:25-43.

Stark SM, Yassa MA, Stark CE (2010) Individual differences in spatial pattern separation performance associated with healthy aging in humans. Learn Mem 17:284-288.

Stark SM, Yassa MA, Lacy JW, Stark CE (2013) A task to assess behavioral pattern separation (BPS) in humans: data from healthy aging and mild cognitive impairment. Neuropsychologia 51:2442-2449.

Stark SM, Stevenson R, Wu C, Rutledge S, Stark CE (2015) Stability of agerelated deficits in the mnemonic similarity task across task variations. Behav Neurosci 129:257-268.

Steffener J, Stern Y (2012) Exploring the neural basis of cognitive reserve in aging. Biochim Biophys Acta 1822:467-473.

Stern Y, Barnes CA, Grady C, Jones RN, Raz N (2019) Brain reserve, cognitive reserve, compensation, and maintenance: operationalization, validity, and mechanisms of cognitive resilience. Neurobiol Aging 83:124129.

Taylor AM, Bus T, Sprengel R, Seeburg PH, Rawlins JN, Bannerman DM (2013) Hippocampal NMDA receptors are important for behavioural 
inhibition but not for encoding associative spatial memories. Philos Trans R Soc Lond B Biol Sci 369:20130149.

Toda T, Gage FH (2018) Review: adult neurogenesis contributes to hippocampal plasticity. Cell Tissue Res 373:693-709.

Toner CK, Pirogovsky E, Kirwan CB, Gilbert PE (2009) Visual object pattern separation deficits in nondemented older adults. Learn Mem 16:338342.

Twarkowski H, Manahan-Vaughan D (2016) Loss of catecholaminergic neuromodulation of persistent forms of hippocampal synaptic plasticity with increasing age. Front Synaptic Neurosci 8:30.

Wu MV, Luna VM, Hen R (2015) Running rescues a fear-based contextual discrimination deficit in aged mice. Front Syst Neurosci 9:114.
Xavier GF, Oliveira-Filho FJ, Santos AM (1999) Dentate gyrus-selective colchicine lesion and disruption of performance in spatial tasks: difficulties in "place strategy" because of a lack of flexibility in the use of environmental cues? Hippocampus 9:668-681.

Yagi S, Chow C, Lieblich SE, Galea LA (2016) Sex and strategy use matters for pattern separation, adult neurogenesis, and immediate early gene expression in the hippocampus. Hippocampus 26:87-101.

Yassa MA, Mattfeld AT, Stark SM, Stark CE (2011) Age-related memory deficits linked to circuit-specific disruptions in the hippocampus. Proc Natl Acad Sci USA 108:8873-8878.

Zeier Z, Madorsky I, Xu Y, Ogle WO, Notterpek L, Foster TC (2011) Gene expression in the hippocampus: regionally specific effects of aging and caloric restriction. Mech Ageing Dev 132:8-19. 